\title{
Hot Electron Effect in Degenerate Semiconductor Tunnel Junction
}

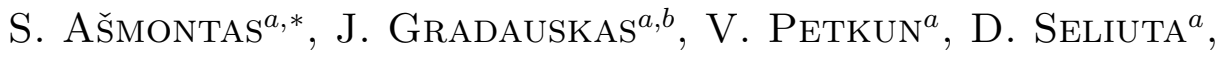
A. SUŽIEDE்LIS ${ }^{a, b}$ AND A. URBELIS ${ }^{b}$

${ }^{a}$ Semiconductor Physics Institute, A. Gotauto 11, 01108 Vilnius, Lithuania

${ }^{b}$ Vilnius Gediminas Technical University, Saultekio 11, 10223 Vilnius, Lithuania

We report on the results of experimental study of free carrier heating in degenerate GaAs tunnel $p-n$ diodes when the carriers are excited by pulsed microwave radiation. Free carrier heating is responsible for the electromotive force in the diode. The magnitude of the electromotive force linearly depends on pulsed microwave power and increases with the decrease in semiconductor lattice temperature. It is almost independent of the pulsed microwave frequency and of $p-n$ junction plane orientation in respect to electric field direction. In the tunnelling regime the dark current in the diode is reduced, however, at high enough forward bias the diffusive current is stimulated due to hot carrier phenomenon.

PACS numbers: 72.20.Ht, 73.40.Gk, 07.57.Kp

\section{Introduction}

Since their invention, the Esaki tunnel $p-n$ diodes, in particular backward ones, are widely used to detect pulsed microwave (MW) radiation [1]. Their operation is based on rectification of high frequency currents due to asymmetry of $I-V$ characteristic.

Due to strong electron-electron and electron-phonon coupling, it appears difficult to heat a degenerate carrier system. For example, free electron heating in metals by electric field in general is ineffective, except special cases, as ultra short excitation by high power pulses or at temperatures below $1 \mathrm{~K}$ [2].

During the last decades free charge carrier heating by radiation in moderately doped semiconductors has been widely investigated at fundamental level as well as for practical purposes of detection of the electromagnetic radiation. A number of investigations revealed that hot carrier electromotive force (emf) $U_{\text {emf }}$ appears under MW and infrared excitation. The emf originates in inhomogeneous semiconductor structures due to a change of carrier mobility and diffusion coefficients under the influence of strong electric field of the radiation (see [3-5], and references

*corresponding author; e-mail: asmontas@pfi2.lt 
therein). In $p-n$ junctions, $U_{\text {emf }}$ may also result from diffusion of heated carriers over the potential barrier. The magnitude of the emf is directly proportional to the potential barrier height $V_{\mathrm{B}}$ of the junction [6]:

$$
U_{\mathrm{emf}}=V_{\mathrm{B}}\left(\frac{T_{\mathrm{e}}}{T}-1\right),
$$

where $T_{\mathrm{e}}$ and $T$ is the electron gas and crystal lattice temperature, respectively. To increase the sensitivity of a diode operating in the carrier heating regime, it is desirable to have a high potential barrier, which, in practice, can be achieved by increasing carrier concentration in the regions close to the junction. But the increase of the doping level leads to degeneration of electron and hole gas, which results in carrier tunneling in the case of an abrupt $p-n$ junction. Thus, the nature of carrier flow across the junction changes, i.e., the tunnel current substitutes for the diffusion current.

In this paper we report our results on free carrier heating by MW radiation in a degenerately doped semiconductor $p-n$ junction. The heating and the measured signal are explained in terms of a classical tunneling model.

\section{Samples and experimental technique}

The tunnel diodes were fabricated from GaAs layers grown by molecular beam epitaxy on semi-insulating GaAs substrates. The thickness of $p$ - and $n$-layer was $400 \mathrm{~nm}$ and $600 \mathrm{~nm}$, respectively. High carrier densities $\left(p=5 \times 10^{19} \mathrm{~cm}^{-3}\right.$ and $n=3 \times 10^{18} \mathrm{~cm}^{-3}$ ) and abrupt doping profile were achieved using amphoteric nature of silicon on (311) GaAs surface. The samples were etched to $120 \times 120 \mu \mathrm{m}^{2}$ mesas with evaporated and annealed $\mathrm{Ni}-\mathrm{Au}-\mathrm{Ge}$ metal contacts on the top.

Magnetron generating $10 \mathrm{~kW}$ at $10 \mathrm{GHz}$ and klystron generating $1 \mathrm{~kW}$ at $35 \mathrm{GHz}$ were used as pulsed $(\sim 1 \mu \mathrm{s})$ MW sources. The diode was placed inside a rectangular waveguide with MW electric field either parallel or perpendicular to the $p-n$ junction plane.

\section{Results and discussion}

The electromotive force induced by MWs across the $p-n$ junction, as Figs. 1 and 2 show, linearly depends on the MW power in a wide range. This is a characteristic feature related to free carrier heating [3].

$U_{\text {emf }}$ depends on lattice temperature $T$ and rises more than twofold as the diode temperature was decreased from room to liquid nitrogen temperature (Fig. 1a). This fact can be explained by a higher value of the difference $T_{\mathrm{e}}-T$ at $T=80 \mathrm{~K}$, i.e. the carriers are relatively stronger heated at lower lattice temperature. Since the value of carrier energy relaxation time increases with the decrease in semiconductor lattice temperature [7], the ratio $\left(T_{\mathrm{e}}-T\right) / T$ is larger at lower $T$ if the strength of the electric field is kept constant (see Eq. (1)).

The dependence of the emf on the incident power at two microwave frequencies is shown in Fig. 1b. A weak dependence of $U_{\text {emf }}$ on frequency evidences that 

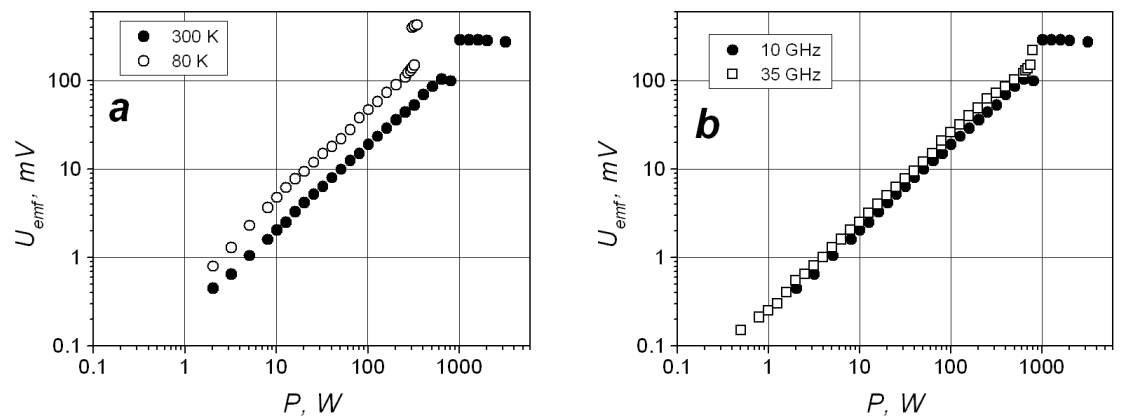

Fig. 1. Emf across the diode versus incident MW power at: (a) room (solid circles) and liquid nitrogen (open circles) temperatures, MW frequency is $10 \mathrm{GHz}$; (b) $10 \mathrm{GHz}$ (solid circles) and $35 \mathrm{GHz}$ (open squares) MW frequencies, room temperature.

the main contribution to $U_{\text {emf }}$ comes from free carrier heating by the electric field of MW radiation, just as it was observed in case of hot carrier thermoelectric force across $n-n^{+}$junction [6].

A similar conclusion can be drawn from the experimental findings of the dependence of $U_{\text {emf }}$ on the diode orientation. A slightly higher value of the emf in the case of the junction plane normal to the direction of incident electric field (Fig. 2) is possibly due to additional rectification of high frequency currents.

To have a better understanding of origin of $U_{\text {emf }}$, an additional bias voltage $U$ was applied to the diode. A complex dependence of the detected signal $U_{\mathrm{d}}$ (voltage across load resistance in series to the diode) on $U$ was observed as shown in Fig. 3 by filled and open circles. The dotted line represents current-voltage
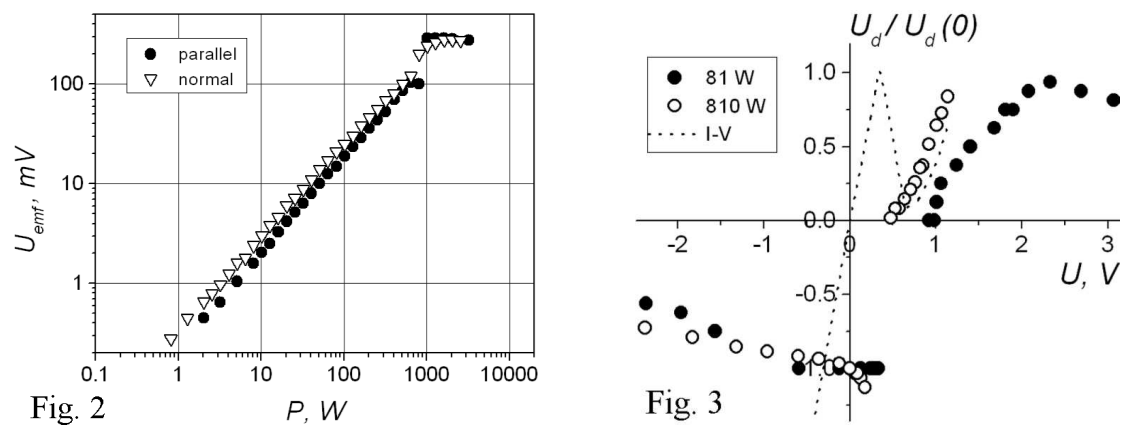

Fig. 2. Emf across the diode versus incident MW power at parallel (solid circles) and normal (open triangles) orientation of the junction plane in respect of electric field direction; room temperature, $10 \mathrm{GHz}$ MW frequency.

Fig. 3. Dependence of detected signal (normalized to zero-bias value) on applied voltage at room temperature. The diode is exposed to the $10 \mathrm{GHz}$ MWs of $81 \mathrm{~W}$ (solid circles) and $810 \mathrm{~W}$ (open circles) of incident power. Dotted line shows current-voltage characteristic of the diode in the dark. 
characteristic of the diode in the dark. Several specific features of the dependence can be distinguished. First, the measured signal monotonically varies with the reverse bias. Second, at $U \approx 0.3 \mathrm{~V}$ the signal abruptly changes its polarity. A similar kind of behaviour of the photocurrent across the tunnel diode was observed when MW radiation was replaced by infrared $\mathrm{CO}_{2}$ laser radiation [8].

At reverse and low forward bias values the dark current flowing across the junction originates from electron tunnelling through the barrier. The tunnel current across the junction can be expressed as [9]

$$
I_{\mathrm{i}}=A \frac{e U}{4 k T}\left(E_{\mathrm{C}}-E_{\mathrm{V}}\right)^{2},
$$

where $A$ is a constant, $E_{\mathrm{C}}$ and $E_{\mathrm{V}}$ represent conduction and valence band edges, respectively, and $k$ is the Boltzmann constant. Polarity of $U_{\mathrm{d}}$ indicates the decrease in dark tunnel current. If one assumes that the degenerate electron and hole gases are heated by MWs to the same temperature $T_{\mathrm{e}}$, then the signal can be expressed as

$$
I_{\mathrm{ph}}=A \frac{e\left[\left(U+U_{\mathrm{emf}}\right) T-U T_{\mathrm{e}}\right]}{4 k T_{\mathrm{e}} T}\left(E_{\mathrm{C}}-E_{\mathrm{V}}\right)^{2},
$$

which is directly proportional to increment of carrier temperature under warm electron condition, when $T_{\mathrm{e}}-T \ll T$.

When the forward bias reaches the negative differential resistance region on $I-V$ characteristic, the dark tunnel current begins to diminish, while the diffusive one rises. This is the reason why at forward bias the abrupt change of $U_{\mathrm{d}}$ polarity takes place. Under these conditions the detected signal is due to heated carrier diffusion over the potential barrier. A further increase in forward bias $U$ causes the measured signal to reach its maximum value, and then to decrease (Fig. 3, solid circles). This kind of behaviour of $U_{\mathrm{d}}$ is characteristic of moderately doped $p-n$ junctions, when the carriers are heated by infrared $[3,8]$ and MW $[6,10]$ radiation. At higher MW power the carriers are heated more intensively, as a result they can diffuse over the barrier at lower forward bias values, i.e. over higher potential barrier (in Fig. 3, the open circles are shifted to the left in regard to solid circles).

One aspect of the voltage-power characteristics in Figs. 1 and 2 should be distinguished. At high enough MW power, $P>800 \mathrm{~W}$ at room temperature, a kind of switching is observed in $U_{\text {emf }}$ : it sharply jumps in magnitude and then levels down to a constant value. Since the polarity of the emf indicates electron flow from $p$ - to $n$-region, a negative charge will be accumulated in the $n$-region. That is, with increased power the diode will be biased in a forward direction. When the induced bias voltage reaches the negative differential resistance region $(\approx 0.3 \mathrm{~V})$, further extra carrier heating does not produce a signal rise. It is worth noting that the voltage-power characteristic remains stable after several measurements over the whole investigated power range. Looking to practical consequences, this 
feature can find application in protection of electronic systems against high power microwave radiation.

\section{Conclusions}

In summary, microwave-induced free carrier heating was experimentally observed in a degenerate GaAs tunnel $p-n$ diode. The following features are revealed:

- emf across the diode is caused by hot carriers, since emf linearly depends on excitation level in a wide range of MW powers; its magnitude grows with a decrease in diode temperature, weakly depends on MW frequency and is insensitive to electric field direction;

- free carrier heating reduces the tunnel current through the diode;

- at forward bias voltages exceeding the negative differential resistance region the signal is induced by hot carrier diffusion over the potential barrier;

- hot carrier effect in an Esaki tunnel $p-n$ diode can be employed to detect MWs as well as to protect electronic systems against high power MW radiation.

\section{Acknowledgments}

The samples were fabricated at Braun Center for Submicron Research at the Weizmann Institute of Sciences under the support of the European Commission within the Large Scale Facility Programme (contract number HPRI-CT-1999-00069).

\section{References}

[1] S.Y. Liao, Microwave Devices and Circuits, 3rd ed., Prentice-Hall, New Jersey 1990, p. 525.

[2] F.C. Wellstood, C. Urbina, J. Clarke, Phys. Rev. B 49, 5942 (1994).

[3] S. Ašmontas, J. Gradauskas, A. Sužiedèlis, E. Širmulis, G. Valušis, Lithuanian J. Phys. 40, 15 (2000).

[4] S. Ašmontas, J. Gradauskas, D. Seliuta, A. Sužiedèlis, E. Širmulis, G. Valušis, J. Thermoelectricity 4, 15 (1998).

[5] A. Sužiedèlis, J. Gradauskas, S. Ašmontas, G. Valušis, H.G. Roskos, J. Appl. Phys. 93, 3034 (2003).

[6] S. Ašmontas, Electrogradient Phenomena in Semiconductors, Mokslas, Vilnius 1984, p. 184.

[7] A. Dargys, J. Kundrotas, Handbook on Physical Properties of Ge, Si, GaAs and InP, Science and Encyclopedia, Vilnius 1994, p. 264.

[8] S. Ašmontas, J. Gradauskas, D. Seliuta, A. Sužiedèlis, G. Valušis, E. Širmulis, in: Proc. Int. Conf. on Advanced Optical Devices, Technologies, and Medical Applications, Eds. J. Spigulis, J. Teteris, M. Ozolinsh, A. Lusis, Proc. SPIE 5123, 221 (2003).

[9] K. Seeger, Semiconductor Physics, Springer-Verlag, Wien 1973, p. 563.

[10] S. Ašmontas, L. Subačius, Lithuanian J. Phys. 15, 789 (1975). 